\title{
Optical Channel Monitoring Technique Using Phase-Modulated Pilot Tones
}

\author{
K. J. Park, H. C. Ji, and Y. C. Chung
}

\begin{abstract}
We report on the pilot-tone-based optical channel monitoring technique for use in wavelength-division-multiplexing (WDM) networks. The proposed technique can monitor the wavelengths of WDM signals using phase-modulated pilot tones. Unlike the previously proposed monitoring technique using amplitude-modulated pilot tones, the proposed technique does not suffer from the cross-gain modulation and stimulated Raman scattering. The results show that the proposed technique could not be deteriorated by the chromatic dispersion.
\end{abstract}

Index Terms-Channel monitoring, phase modulation, pilot tone, wavelength.

\section{INTRODUCTION}

$\mathbf{R}$ ECENTLY, there have been many efforts to use pilot tones (i.e., small sinusoidal components added to wavelengthdivision-multiplexing (WDM) signals) for the monitoring of WDM signals directly in the optical layer. For example, it has been reported that the pilot tone could be used to monitor various optical parameters of WDM signals such as wavelength, optical power, chromatic dispersion, polarization-mode dispersion, and optical signal-to-noise ratio, etc. [1]-[4]. The pilot-tone-based techniques can monitor these parameters without using tunable filters. Thus, this technique can be cost-effective. In addition, this technique is well suited for the use in a dynamic WDM network, since the pilot tones are bound to follow their corresponding optical signals wherever in the network. Thus, the optical path of each WDM signal could be monitored simply by tracking its tone frequency [5]. Although the conventional monitoring technique based on amplitude-modulated (AM) pilot tones has many advantages, it also has some limitations such as receiver sensitivity degradation and "ghost tones" [6], [7]. To mitigate these problems, we have previously proposed the monitoring technique based on frequency-modulated (FM) pilot tones [8]. However, this technique has two critical problems for the practical use in a WDM network. First, it should be required to use an additional intensity modulator to cancel out the undesirable AM component. Thus, because the operating bias condition of conventional intensity modulator can be changed due to the temperature variation, it is difficult to generate a long-stable pure FM pilot tone. Second, this technique utilizes the current dithering to

Manuscript received January 24, 2005; revised July 15, 2005.

K. J. Park is with the Department of Electrical Engineering, Korea Advanced Institute of Science and Technology, Daejeon 305-701, Korea, and also with the Digital Solution Center, Samsung Electronics, Seoul 135-524, Korea (e-mail: k.j.park@samsung.com).

H. C. Ji and Y. C. Chung are with the Department of Electrical Engineering, Korea Advanced Institute of Science and Technology, Daejeon 305-701, Korea. Digital Object Identifier 10.1109/LPT.2005.857243

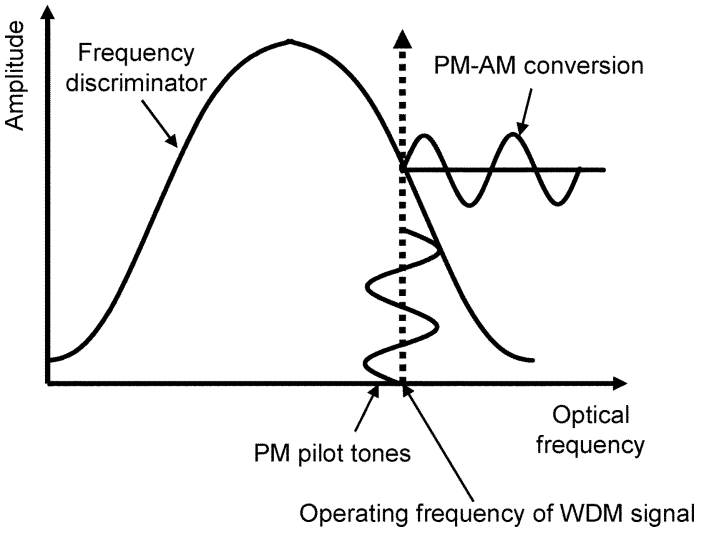

Fig. 1. Operating principle of the proposed technique.

generate the FM modulation. Thus, because the tone frequency should be less than $20 \mathrm{kHz}$ due to the thermal modulation characteristics of distributed feedback (DFB) laser [9], it is also difficult to support a large number of pilot tones (i.e., WDM channels). In this letter, we propose and demonstrate a novel optical channel monitoring technique using phase-modulated (PM) pilot tones. A long-stable pure PM pilot tone can be generated simply by using a phase modulator. In addition, the proposed technique can support a large number of pilot tones since it utilizes high-frequency pilot tones $(>100 \mathrm{MHz})$. It should be noted that, the proposed technique using PM pilot tones could overcome the problems inherent in the monitoring technique based on the AM pilot tones, while it maintains all the advantages of using pilot tones. However, we have recently pointed out that the undesirable AM component can be generated due to the chromatic dispersion when the phase-modulated optical signal is propagated through an optical fiber [10]. Thus, this AM component may cause a monitoring error. To solve this problem, we have found that the tone frequency should be less than $1 \mathrm{GHz}$. The results show that the proposed technique can monitor the optical paths, wavelengths, and powers of WDM signals without any performance degradation.

\section{OPERATING PRINCIPLE}

Fig. 1 shows the operating principle of the proposed monitoring technique using a PM pilot tone. The optical phase of the laser including the PM pilot tone $\phi(t)$ can be described as

$$
\phi(t)=\phi_{0}+\Delta \phi \sin (\omega t)
$$

where $\phi_{0}$ and $\Delta \phi$ are the operating phase and the peak phase deviations of the laser, respectively, and $\omega$ is the angular modulation frequency of the pilot tone. When this PM pilot tone passes through the frequency discriminator, the amplitude of the optical 


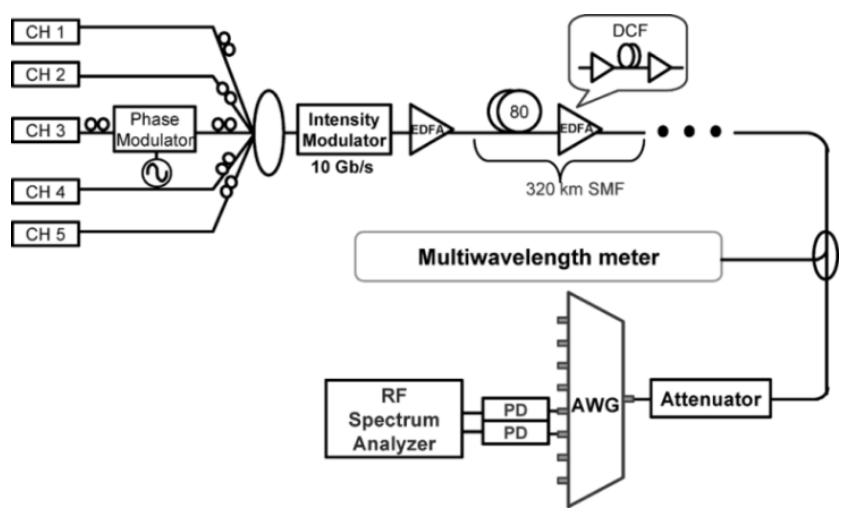

Fig. 2. Experimental setup.

signal becomes to be modulated due to the PM-AM conversion, as shown in Fig. 1 [11]. In this case, the magnitude of AM component $\Delta P$ can be expressed as

$$
\Delta P \propto \frac{T\left(\nu_{0}\right) T^{\prime}\left(\nu_{0}\right) \Delta \phi \omega}{2 \pi}
$$

where $T(\nu)$ and $T^{\prime}(\nu)$ represent the transmission function of the frequency discriminator and its first derivative, respectively. Equation (2) indicates that the AM component has different magnitude according to the operating frequency of WDM signal. Thus, the operating frequency of WDM signal can be easily monitored by measuring the magnitude of AM component. It should be noted that, in the proposed monitoring technique using PM pilot tones, the ghost tones caused by cross-gain modulation (XGM) of erbium-doped fiber amplifier (EDFA) and stimulated Raman scattering (SRS) of an optical fiber can not be generated since the magnitude of WDM signal is maintained to be constant along the transmission line [7]. In addition, unlike the monitoring technique using AM pilot tones, the performance of WDM signal can not be deteriorated due to the interference with the pilot tone.

\section{EXPERIMENTS AND RESULTS}

Fig. 2 shows the experimental setup used to demonstrate the principle of the proposed monitoring technique. We used five DFB lasers operating at $192.4 \sim 193.2 \mathrm{THz}$ (channel spacing $=200 \mathrm{GHz}$ ). The optical phase of one DFB laser (Channel 3) was modulated by using a $\mathrm{LiNbO}_{3}$ phase modulator. All WDM signals were combined and modulated at $10 \mathrm{~Gb} / \mathrm{s}$ (pattern length: $2^{31}-1$ ) using a high-speed $\mathrm{LiNbO}_{3}$ modulator, and then sent to the arrayed waveguide grating (AWG) via EDFA chain for the optical channel monitoring (AWG input power $=3 \mathrm{dBm}$ ). Fig. 3 shows the amplitudes of AM components (tones) of Channel 3 and the ratio between these amplitudes measured at two adjacent AWG ports while varying the optical frequency (tone frequency $=504 \mathrm{MHz}$ and peak phase deviation $=0.5 \pi$ ). In this figure, "A" represents the amplitude of AM component measured at the third port of AWG, while "B" represents the amplitude obtained at the fourth port. These AM components are proportional to the transmission function of AWG as expressed in (2). The result shows that the ratio curve is nearly linear $(\sim 0.4 \mathrm{~dB} / \mathrm{GHz})$ with the operating frequency of the optical signal. It should be

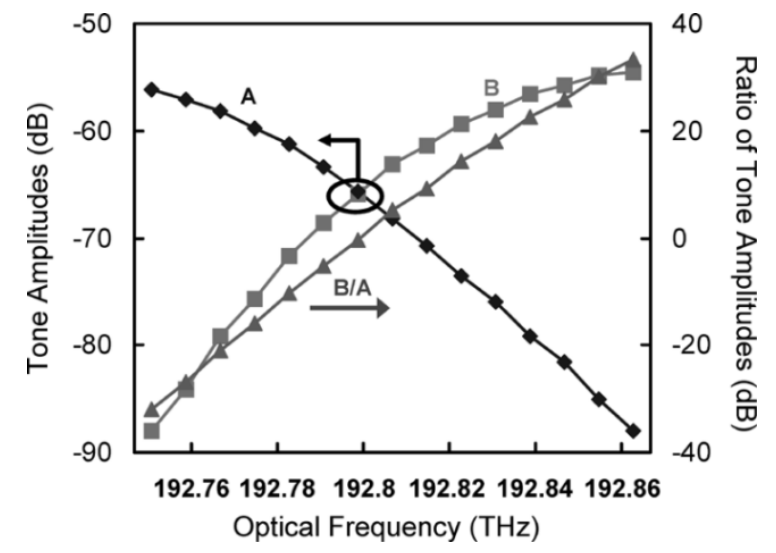

Fig. 3. Amplitudes and ratio of AM components measured at two adjacent AWG ports.

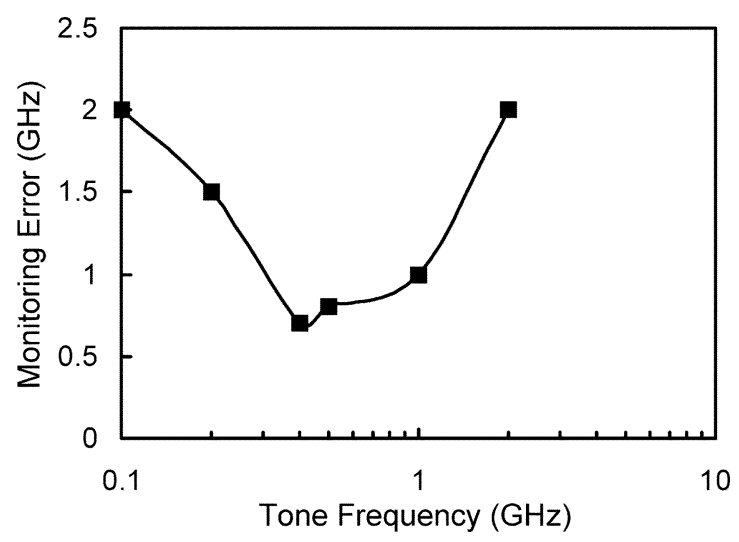

Fig. 4. Frequency monitoring error measured while varying the tone frequency (accumulated dispersion $=1000 \mathrm{ps} / \mathrm{nm}$ ) when the WDM channel was operating at $192.8 \mathrm{THz}$.

noted that the crossover points of the AWG were anchored at the ITU-standardized frequencies by using a temperature controller [12]. Thus, the operating frequencies of WDM signals can be estimated by measuring the ratios of tone amplitudes at the corresponding tone frequencies and AWG ports. In addition, the optical power of each WDM channel can also be monitored easily by measuring their corresponding tone amplitudes. However, (2) indicates that if the tone frequency were too low, the magnitude of the AM components would not be measured. The monitoring sensitivity of this technique could be improved by increasing the tone frequency, but it would deteriorate the accuracy due to the PM-AM conversion caused by the chromatic dispersion [10]. Thus, the tone frequency should be optimized to obtain the best performance. Fig. 4 shows the monitoring error measured while varying the tone frequency (accumulated dispersion $=1000 \mathrm{ps} / \mathrm{nm}$ ) when the WDM channel was operating at $192.8 \mathrm{THz}$. The results show that the monitoring error increases when the tone frequency is lower than $200 \mathrm{MHz}$. This is mainly because the magnitude of the AM components is too small to be measured accurately (RF power: $<-73 \mathrm{dBm}$, carrier-to-noise ratio: $<10 \mathrm{~dB}$, resolution bandwidth $=50 \mathrm{kHz}$ ). It should be noted that these results would not be changed even when the number of WDM channels is higher than five since the proposed technique detected only two WDM channels per photodetector (the WDM 


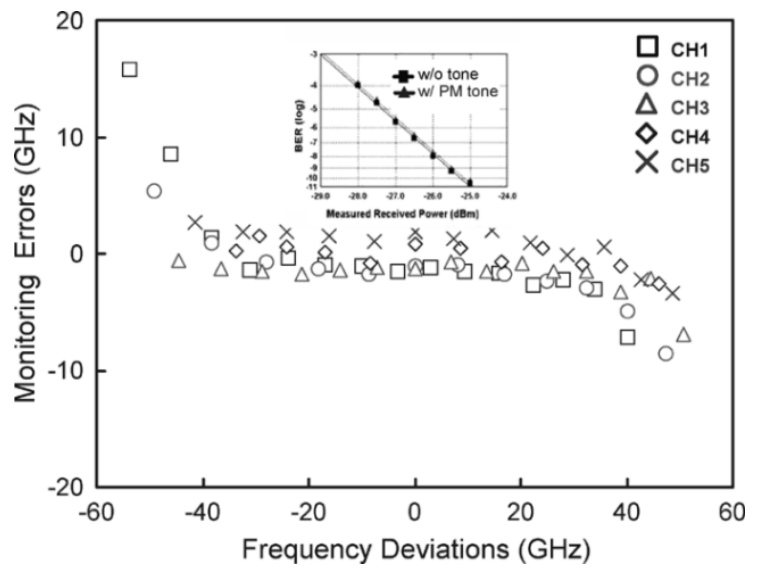

Fig. 5. Frequency monitoring errors measured after the transmission of $10-\mathrm{Gb} / \mathrm{s}$ signals over $320 \mathrm{~km}$ of SMF. The inset shows the BER curve measured when a PM pilot tone was added to the optical signal.

channels are demultiplexed before the detection). On the other hand, when the tone frequency is higher than $1 \mathrm{GHz}$, the monitoring error is also increased by the chromatic dispersion. Thus, we found that the frequency of PM pilot tone should be about $500 \mathrm{MHz}$ for the accurate optical channel monitoring.

Fig. 5 shows the frequency monitoring errors measured after the transmission of $10-\mathrm{Gb} / \mathrm{s}$ signals over $320 \mathrm{~km}$ of dispersion-managed single-mode fiber (SMF) using $\mathrm{PM}$ pilot tones (SMF launch power $=3 \mathrm{dBm}$ and DCF launch power $=-3 \mathrm{dBm}$ ). In the experiment, the optical phases of the lasers were modulated at $500 \sim 508 \mathrm{MHz}$ (peak deviation $=0.5 \pi$ ) with a channel separation of $2 \mathrm{MHz}$. The horizontal axis represents the optical frequency deviation from the ITU-standardized frequency of each optical channel. In this measurement, we intentionally changed the optical frequency of each laser up to $\pm 50 \mathrm{GHz}$ using a temperature controller. The optical frequencies of five WDM channels could be measured with accuracy better than $\pm 3 \mathrm{GHz}$, when the WDM lasers were operating within $\pm 40 \mathrm{GHz}$ from the ITU-standardized frequencies. The monitoring errors were mainly attributed to the uneven transmission characteristics of AWG. When phase-modulated optical signals propagate through an optical amplifier and an optical filter, undesirable AM components can be generated due to the PM-AM conversion. However, the performance of the proposed technique cannot be deteriorated because the transmission characteristics of the conventional optical amplifier and optical filter have nearly flat responses at the operating frequencies (for example, the typical transmission slopes of our optical amplifiers and optical filters were less than 0.001 and $0.01 \mathrm{~dB} / \mathrm{GHz}$, respectively). Thus, we did not observe any significant AM components due to PM-AM conversion after the transmission over $320-\mathrm{km}$ link (including five two-stage EDFAs and one optical filter). It should be noted that the performance of the proposed monitoring technique could not be deteriorated due to the PM-AM conversion caused by chromatic dispersion after the transmission over 320-km SMF (residual dispersion $=200 \mathrm{ps} / \mathrm{nm}$ ) by using the optimum tone frequencies. Moreover, we have recently pointed out that the effects of ghost tones caused by XGM and SRS can be neglected in a 64-channel WDM network (transmission distance $=640 \mathrm{~km}$ ) when the tone frequencies are higher than $100 \mathrm{MHz}$ [7]. Thus, using the proposed technique, the optical path of each WDM channel can be monitored anywhere in the network simply by tracking their corresponding tone frequencies since every laser (or channel) is assigned with a unique tone frequency. In particular, the inset shows the bit-error-rate (BER) curve measured when a PM pilot tone was added to the optical signal. These results confirm that the PM pilot tone does not degrade the receiver sensitivity.

\section{SUMMARY}

We demonstrated a simple technique that can be used to monitor the wavelengths of optical signals. The proposed technique based on PM pilot tones could monitor the wavelengths (optical frequencies) of WDM signals with accuracy better than $\pm 3 \mathrm{GHz}$ even after the transmission over $320 \mathrm{~km}$ of SMF. The proposed technique does not suffer from the problems (such as ghost tones caused by XGM and SRS, and tone-induced receiver sensitivity degradation) of the conventional pilot-tone-based monitoring technique, while it maintains all the advantages of using pilot tones.

\section{REFERENCES}

[1] K. J. Park, S. K. Shin, and Y. C. Chung, "Simple monitoring technique for WDM networks,” Electron. Lett., vol. 35, pp. 415-417, 1999.

[2] M. N. Petersen, Z. Pan, S. Lee, S. A. Havstad, and A. E. Willner, "Online chromatic dispersion monitoring and compensation using a single inband subcarrier tone," IEEE Photon. Technol. Lett, vol. 14, no. 4, pp. 570-572, Apr. 2002.

[3] S. M. R. Motaghian Nezam, Y. Wang, M. Hauer, S. Lee, and A. E. Willner, "Simultaneous PMD monitoring of several WDM channels using subcarrier tones," in Conf. Lasers and Electro-Optics (CLEO 2001), 2001, Paper CFE1, pp. 561-562.

[4] G. Rossi, T. E. Dimmick, and D. J. Blumenthal, "Optical performance monitoring in reconfigurable WDM optical networks using subcarrier multiplexing," J. Lightw. Technol, vol. 18, no. 12, pp. 1639-1648, Dec. 2000.

[5] G. R. Hill, P. J. Chidgey, F. Kaufhold, T. Lynch, O. Sahlen, M. Gustavsson, B. Lagerstrom, G. Grasso, F. Meli, S. Johansson, J. Ingers, L. Fernandez, S. Rotolo, A. Antonielli, S. Tebaldini, E. Vezzoni, R. Caddedu, N. Caponio, F. Testa, A. Scavennec, M. J. O’Mahony, J. Zhou, A. Yu, W. Sohler, U. Rust, and H. Herrmann, "A transport network layer based on optical network elements," J. Lightw. Technol, vol. 11, no. 5, pp. 667-679, May/Jun. 1993.

[6] M. Murakami, T. Imai, and M. Aoyama, "A remote supervisory system based on subcarrier overmodulation for submarine optical amplifier systems," J. Lightw. Technol, vol. 14, no. 5, pp. 671-677, May 1996.

[7] H. S. Chung, S. K. Shin, K. J. Park, H. G. Woo, and Y. C. Chung, "Effects of stimulated Raman scattering on pilot-tone-based WDM supervisory technique," IEEE Photon. Technol. Lett, vol. 12, no. 6, pp. 731-733, Jun. 2000.

[8] K. J. Park, C. J. Youn, J. H. Lee, and Y. C. Chung, "Optical path, wavelength, and power monitoring technique using frequency-modulated pilot tones," in Tech. Dig. OFC 2004, Los Angeles, CA, Feb. 2004 Paper FF1.

[9] D. Welford and S. B. Alexander, "Magnitude and phase characteristics of frequency modulation in directly modulated GaAlAs semiconductor diode lasers," J. Lightw. Technol, vol. LT-3, no. 5, pp. 1092-1099, Oct. 1985.

[10] K. J. Park, C. J. Youn, J. H. Lee, and Y. C. Chung, "Performance comparisons of chromatic dispersion-monitoring techniques using pilot tones,' IEEE Photon. Technol. Lett., vol. 15, no. 6, pp. 873-875, Jun. 2003.

[11] M. R. Phillips and D. M. Ott, "Crosstalk caused by nonideal output filters in WDM lightwave systems," IEEE Photon. Technol. Lett., vol. 12, no. 8, pp. 1094-1096, Aug. 2000.

[12] C. J. Youn, S. K. Shin, K. J. Park, and Y. C. Chung, "Optical frequency monitoring technique using arrayed waveguide grating and pilot tones,' Electron. Lett, vol. 37, no. 16, pp. 1032-1033, 2001. 